\title{
Thermoelectric properties of p-type filled skutterudites $\mathrm{S}_{\mathrm{x}} \mathrm{Co}_{4} \mathrm{Sb}_{12}$
}

Cite as: AIP Conference Proceedings 2265, 030606 (2020); https://doi.org/10.1063/5.0016889 Published Online: 05 November 2020

Sanyukta Ghosh, Krushna Kumari Raut, Anbalagan Ramakrishnan, Kuei-Hsien Chen, Soon-Jik Hong, and Ramesh Chandra Mallik

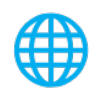

\section{ARTICLES YOU MAY BE INTERESTED IN}

Facile and fast, microwave assisted solvothermal synthesis of $\mathrm{Sb}_{2} \mathrm{Se}_{3}$ nanostructures for thermoelectric applications

AIP Conference Proceedings 2265, 030144 (2020); https://doi.org/10.1063/5.0017553

Near room temperature thermoelectrics: $\mathrm{Ag}_{2} \mathrm{Se}$

AIP Conference Proceedings 2265, 030429 (2020); https://doi.org/10.1063/5.0017451

Enhanced thermoelectric properties of $\mathrm{Hf}$ doped half-Heusler compound $\mathrm{NbFeSb}$

AIP Conference Proceedings 2265, 030459 (2020); https://doi.org/10.1063/5.0017704

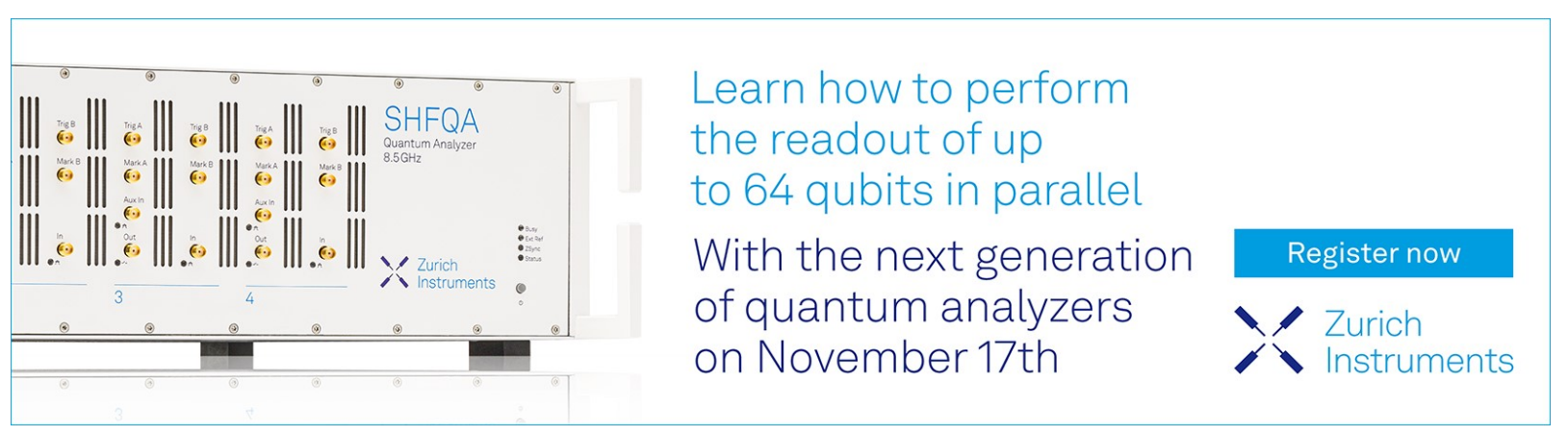




\title{
Thermoelectric Properties of p-type Filled Skutterudites $\mathrm{S}_{\mathrm{x}} \mathrm{Co}_{4} \mathrm{Sb}_{12}$
}

\author{
Sanyukta Ghosh ${ }^{1}$, Krushna Kumari Raut ${ }^{1}$, Anbalagan Ramakrishnan ${ }^{2}$, Kuei-Hsien \\ Chen $^{2}$, Soon-Jik Hong ${ }^{3}$ and Ramesh Chandra Mallik ${ }^{1,3, a)}$ \\ ${ }^{1}$ Thermoelectric Materials and Devices Laboratory, Department of Physics, Indian Institute of Science, Bangalore \\ 560012, India. \\ ${ }^{2}$ Institute of Atomic and Molecular Sciences, Academia Sinica, No. 1, Sec. 4, Roosevelt Road, Taipei, Taiwan. \\ ${ }^{3}$ Division of Advanced Materials Engineering, 275, Budae-dong, Cheonan, Chungnam, 330-71, Kongju National \\ University, Republic of Korea. \\ ${ }^{a)}$ Corresponding author: rcmallik@iisc.ac.in
}

\begin{abstract}
The electronegativity difference between the filler and the host atom of skutterudites determines the bonding type, which effects the thermoelectric properties. The electropositive elements with low electronegativity compared to $\mathrm{Sb}$ atom can form n-type filled skutterudites. In this study, the electronegative element sulphur has filled into voids. A series of $\mathrm{S}_{x} \mathrm{Co}_{4} \mathrm{Sb}_{12}(\mathrm{x}=0.05$ to 0.4$)$ compounds were synthesized by the solid-state method. CoSbS secondary phase was observed for $x>0.05$. Filling fraction of sulphur into the void $(-0.04)$ was calculated using electron probe microanalyzer which agrees with Rietveld refinement of the X-ray diffraction pattern. The transport properties were measured from 350 $\mathrm{K}$ to $723 \mathrm{~K}$. The positive Seebeck coefficient (S) confirms p-type semiconducting nature. S and the electrical resistivity $(\rho)$ decreased with increasing $x$ due to increased carrier concentration (n). All the samples except $x=0.05$ possess almost same $\mathrm{S}$ and $\rho$ due to saturation of filling fraction. The enhanced phonon scattering in $\mathrm{S}_{x} \mathrm{Co}_{4} \mathrm{Sb}_{12}$ resulted in low thermal conductivity $(\kappa)$. The highest zT of 0.18 at $623 \mathrm{~K}$ was achieved for $\mathrm{S}_{0.15} \mathrm{Co}_{4} \mathrm{Sb}_{12}$.
\end{abstract}

\section{INTRODUCTION}

Skutterudite materials have shown efficient performance at a mid-temperature range between $500 \mathrm{~K}$ to $800 \mathrm{~K}$ due to its beneficial properties such as highly symmetric bcc structure, high Seebeck coefficient (S), low electrical resistivity $(\rho)$, the covalent bond between $\mathrm{Co}$ and $\mathrm{Sb}$ and high mechanical stability [1]. But high thermal conductivity ( $\kappa)$ of $\mathrm{Co}_{4} \mathrm{Sb}_{12}\left(8 \mathrm{~W} / \mathrm{m}-\mathrm{K}\right.$ at $300 \mathrm{~K}$ [2]) leads to a low figure of merit ( $\left.\mathrm{zT}=\mathrm{S}^{2} \mathrm{~T} / \mathrm{\rho} \kappa\right)$. Incorporation of suitable foreign elements into the voids of skutterudite structures leads to low $\kappa$ and high zT. According to Shi et al., only the electropositive fillers which satisfy this selection rule: $\chi_{s b}-\chi_{f}>0.80$ have negative formation enthalpy and can form heavily doped n-type filled skutterudites, where $\chi_{S b}$ and $\chi_{f}$ are the electronegativities of $\mathrm{Sb}$ atom and fillers, respectively [3]. The difference in electronegativity between the filler and the host atom determines the chemical bonding type, which influences the thermoelectric transport properties. Recently several research papers have published on electronegative element filled $\mathrm{Co}_{4} \mathrm{Sb}_{12}$, which has a higher electronegativity compared to $\mathrm{Sb}$ [4]. These efforts will allow developing n-type and p-type materials by filling both electropositive and electronegative elements simultaneously. The electronegative fillers were incorporated into the host structure along with other $\mathrm{n}$ type dopants at the $\mathrm{Co} / \mathrm{Sb}$ sites [4,5] or using non-equilibrium synthesis method [6].

In this study, the electronegative element sulphur has been used as a filler into the skutterudite voids. $\mathrm{S}_{\mathrm{X}} \mathrm{Co}_{4} \mathrm{Sb}_{12}$ ( $\mathrm{x}=0.05$ to 0.4 ) compounds were prepared by solid-state method and densified using hot press sintering. The filling fraction limit (FFL) of sulphur into the voids was around 0.04. The carrier concentration (n) increased with increasing sulphur content. As a result, $\rho$ decreased, and $\mathrm{S}$ increased with increasing $\mathrm{x}$. An increase in power factor 
(P.F) and decrease in $\kappa$ led to improved zT of 0.18 at $623 \mathrm{~K}$ for $\mathrm{S}_{0.15} \mathrm{Co}_{4} \mathrm{Sb}_{12}$, which is higher than the pristine $\mathrm{Co}_{4} \mathrm{Sb}_{12}$. In this work, we mainly focus on the synthesis of $\mathrm{p}$-type electronegative guest-filled $\mathrm{Co}_{4} \mathrm{Sb}_{12}$.

\section{EXPERIMENTAL DETAILS}

High purity elements Co (99.995\%), Sb (99.999\%) and S (99.999\%) were weighted according to the stoichiometric ratio and were mixed and ground into a fine powder using mortar and pestle. The powder was coldpressed to form pellets. The cold-pressed pellets were sealed in the quartz ampoule under a vacuum of $10^{-4} \mathrm{mbar}$ and heat-treated at $953 \mathrm{~K}$ for 60 hours. The heat-treated samples were reground and hot-pressed at $923 \mathrm{~K}$ under a pressure of $35 \mathrm{mPa}$ for 2 hours. The density (d) of the hot-pressed samples were determined using Archimedes' principle and were $>90 \%$ of the theoretical density. The X-ray diffraction pattern (XRD) of the powder samples was carried out by Rigaku SmartLab diffraction system using $\mathrm{Cu} \mathrm{K} \mathrm{K}_{\alpha}$ source $(\lambda=1.5406 \AA)$. The Rietveld refinement analysis of the XRD pattern was done to find put the lattice parameter and sulphur content in the sample. FEI ESEM Quanta 200 was used to observe the surface morphology of all the samples. The composition of the samples was obtained using wavelength dispersive spectroscopy (WDS) attached with an EPMA, JEOL JXA-8530F. The Hall measurement at room temperature was carried out to calculate the carrier concentration of the samples using a magnetic field of $0.55 \mathrm{~T}$. The rectangular pellets of dimensions $12 \times 2 \times 2 \mathrm{~mm}^{3}$ were used to measure the Seebeck coefficient and electrical resistivity. The Seebeck coefficient and electrical resistivity were measured using Linseis LSR-3 from $350 \mathrm{~K}$ to $750 \mathrm{~K}$. The samples were cut into cylindrical pellets of $6 \mathrm{~mm}$ diameter with a thickness of 1 $\mathrm{mm}$ to measure the thermal diffusivity. The thermal diffusivity (D) and specific heat $\left(\mathrm{C}_{\mathrm{p}}\right)$ were measured from 350 $\mathrm{K}$ to $750 \mathrm{~K}$ using a Netzsch LFA 457 laser flash equipment. The total thermal conductivity was calculated using the formula $\kappa=D C_{p} d$. The errors in the S, $\rho$ and $\kappa$ measurement are $7 \%, 10 \%$ and $6 \%$ respectively.

\section{RESULTS AND DISCUSSIONS}

The XRD pattern of $\mathrm{S}_{\mathrm{x}} \mathrm{Co}_{4} \mathrm{Sb}_{12}$ where $\mathrm{x}=0.05,0.1,0.15,0.2,0.25,0.3$ and 0.4 is shown in Fig. 1(a). All the peaks in the XRD pattern were indexed with skutterudite phase according to ICSD\#41620. No shift in the peak was observed with increasing $\mathrm{x}$, which could be due to the very low amount of sulphur into the voids. This was further validated from Rietveld refinement analysis of the XRD pattern, which showed no change in the lattice parameter with the change in $\mathrm{x}$ (table 1). The Rietveld refinement analysis of the XRD pattern of $\mathrm{S}_{0.15} \mathrm{Co}_{4} \mathrm{Sb}_{12}$ is shown in Fig. 1(b). The composition evaluated using EPMA analysis was considered in the Rietveld refinement, which exhibited good fitting.

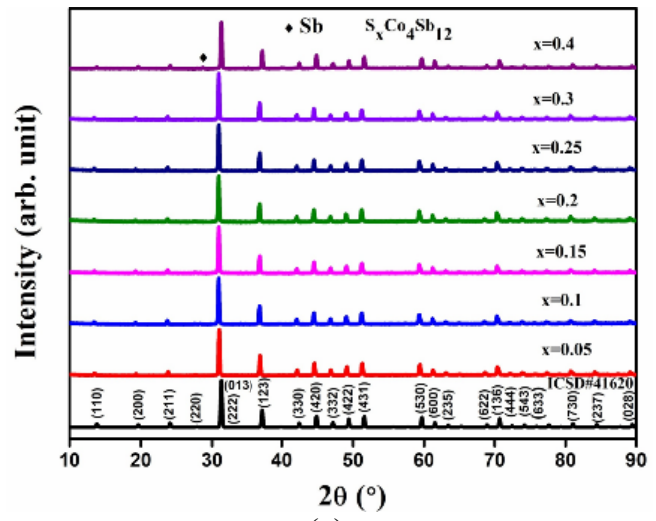

(a)

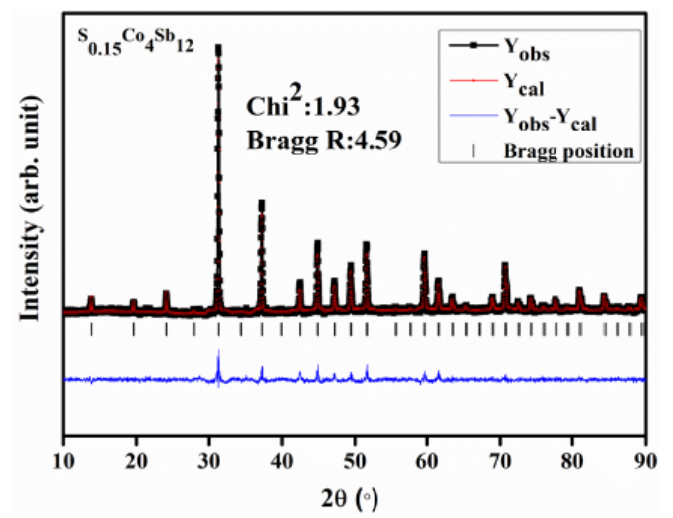

(b)

FIGURE 1. (a) Powder XRD patterns of $\mathrm{S}_{\mathrm{x}} \mathrm{Co}_{4} \mathrm{Sb}_{12}$ (where $\mathrm{x}=0.05,0.1,0.15,0.2,0.25,0.3$ and 0.4 ), (b) Rietveld refinement analysis of XRD pattern of $\mathrm{S}_{0.15} \mathrm{Co}_{4} \mathrm{Sb}_{12}$. 
TABLE 1. Lattice parameter, band gap and carrier concentration of $\mathrm{S}_{\mathrm{x}} \mathrm{Co}_{4} \mathrm{Sb}_{12}$ at room temperature.

\begin{tabular}{cccc}
\hline $\begin{array}{c}\text { Sample } \\
\mathrm{S}_{\mathrm{x}} \mathrm{Co}_{4} \mathrm{Sb}_{12}\end{array}$ & Lattice parameter $(\AA)$ & $\begin{array}{c}\text { Band gap } \\
\left(\mathrm{E}_{\mathrm{g}}=2 \mathrm{~S}_{\max } \mathrm{T}_{\max }\right)[7] \mathrm{in} \mathrm{eV}\end{array}$ & $\begin{array}{c}\text { Carrier concentration } \\
\mathrm{cm}^{-3}(\mathrm{n})\end{array}$ \\
\hline $\mathrm{x}=0.05$ & 9.032 & 0.24 & $7.78 \times 10^{17}$ \\
$\mathrm{x}=0.1$ & 9.031 & 0.19 & $7.84 \times 10^{18}$ \\
$\mathrm{x}=0.15$ & 9.032 & 0.19 & $8.77 \times 10^{18}$ \\
$\mathrm{x}=0.2$ & 9.032 & 0.19 & $8.67 \times 10^{18}$ \\
$\mathrm{x}=0.25$ & 9.031 & 0.19 & $8.12 \times 10^{18}$ \\
$\mathrm{x}=0.3$ & 9.031 & 0.19 & $8.06 \times 10^{18}$ \\
$\mathrm{x}=0.4$ & 9.030 & 0.18 & $1.11 \times 10^{19}$ \\
\hline
\end{tabular}

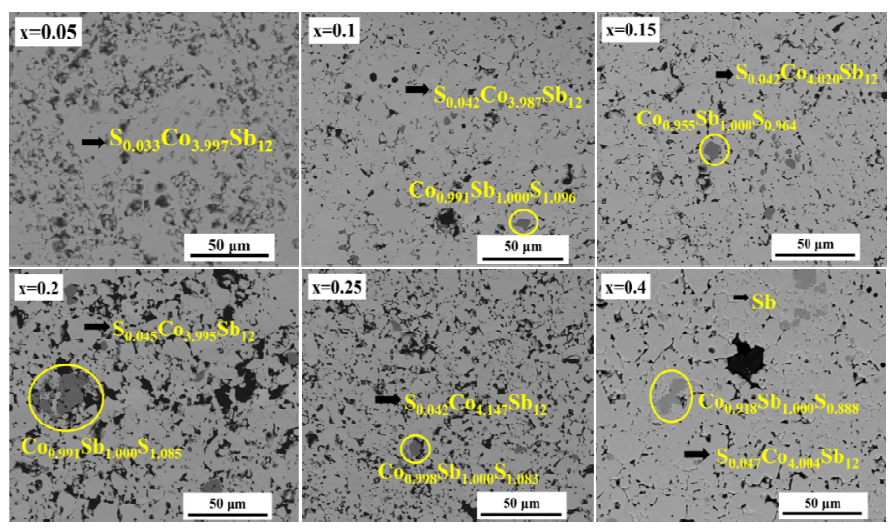

FIGURE 2. $\mathrm{SEM}$ micrographs of $\mathrm{S}_{\mathrm{x}} \mathrm{Co}_{4} \mathrm{Sb}_{12}$.

Fig. 2 shows the backscattered electron (BSE) micrographs of $\mathrm{S}_{\mathrm{x}} \mathrm{Co}_{4} \mathrm{Sb}_{12}$. The impurity phase of CoSbS was observed for a higher content of sulphur (for $\mathrm{x}>0.05$ ) in the sample. An additional secondary phase of Sb was observed for the sample with $\mathrm{x}=0.4$. The composition of the main phase and secondary phases were determined using EPMA, which showed the maximum filling fraction of 0.04 of sulphur into the voids. Fig. 3 (a-f) shows the temperature dependence of the thermoelectric properties. The positive Seebeck coefficient (S) indicates holes as the majority charge carrier for all the samples. S increased with increasing temperature up to $600 \mathrm{~K}$, and then it decreased at the higher temperature. The electrical resistivity $(\rho)$ decreased with increasing temperature, which indicates semiconducting behaviour of all the samples. A decrease in $\mathrm{S}$ and $\rho$ was observed with increasing $\mathrm{x}$. This is because sulphur donated holes to the network and increased the carrier concentration with increasing $\mathrm{x}$, which was confirmed from the Hall measurement (table 1). All the samples with higher sulphur content $(\mathrm{x}>0.05)$ possess almost the same $\mathrm{S}$ and $\rho$ due to saturation of the filling fraction of sulphur. The band gap of the samples was calculated using the equation given by Goldsmid and Sharp [7] which is listed in table 1. The saturation of the sulphur content also resulted in same band gap value for the samples with $\mathrm{x}>0.05$. $\mathrm{S}$ and $\rho$ are mostly affected by the impurity phase CoSbS for $\mathrm{x}>0.05$. The maximum power factor (P.F) of $10.9 \mu \mathrm{w} / \mathrm{cm}^{-K^{2}}$ at $540 \mathrm{~K}$ was achieved for the sample with $\mathrm{x}=0.15$ due to low electrical resistivity. The total thermal conductivity $(\kappa)$ decreased with increasing temperature for all the samples following 1/T law. Therefore, Umklapp scattering is the main phonon scattering mechanism. $\kappa$ is composed of lattice part of thermal conductivity $\left(\kappa_{\mathrm{L}}\right)$ and electronic part of thermal conductivity $\left(\kappa_{\mathrm{e}}\right) ; \kappa=\kappa_{e}+\kappa_{L}$. $\kappa_{\mathrm{e}}$ was evaluated using the Wiedemann-Franz relation, $\kappa_{e}=L T / \rho$ where $\mathrm{L}$ is the temperature-dependent Lorenz number. $\kappa_{\mathrm{L}}$ was calculated by separating $\kappa_{\mathrm{e}}$ from $\kappa\left(\kappa_{L}=\kappa-\kappa_{e}\right)$. $\kappa_{\mathrm{L}}$ also decreased with increasing temperature for all the samples. $\kappa$ decreased in $\mathrm{S}_{\mathrm{x}} \mathrm{Co}_{4} \mathrm{Sb}_{12}$ compared to the pristine sample, which is 
due to the enhanced phonon scattering in the filled skutterudite. The lowest $\kappa$ of $2.72 \mathrm{~W} / \mathrm{m}-\mathrm{K}$ at $623 \mathrm{~K}$ was obtained in the sample with $\mathrm{x}=0.05$. The slight difference in $\kappa_{\mathrm{L}}$ for the samples with $\mathrm{x}>0.05$ is mostly coming from the impurity phase. $\mathrm{S}_{0.15} \mathrm{Co}_{4} \mathrm{Sb}_{12}$ compound exhibited the maximum figure of merit (zT) of 0.18 at $623 \mathrm{~K}$ due to comparatively high P.F and low $\kappa$.

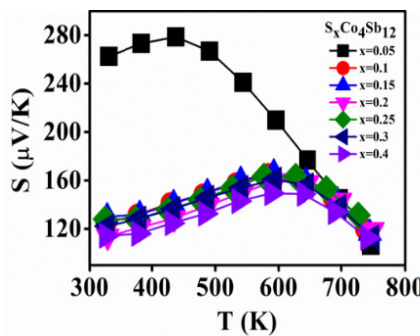

(a)

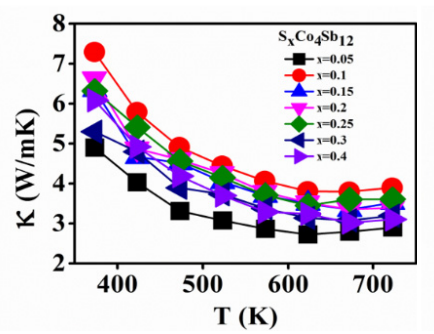

(d)

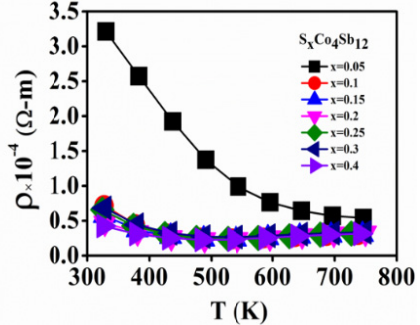

(b)

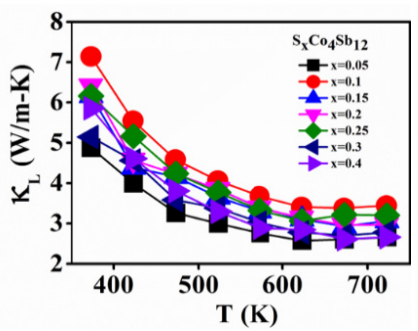

(e)

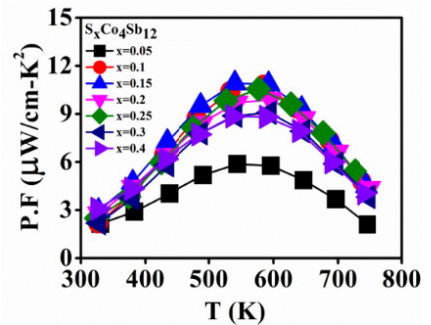

(c)

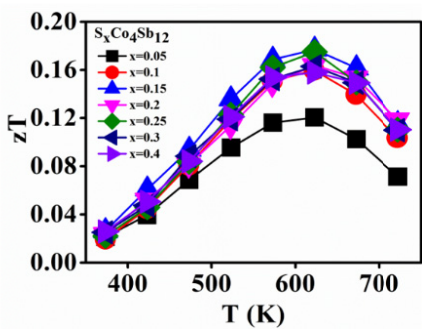

(f)

FIGURE 3. (a) The Seebeck coefficient (S), (b) the electrical resistivity ( $\rho)$, (c) the power factor (P.F), (d) the total thermal conductivity $(\kappa)$, (e) the lattice thermal conductivity $\left(\kappa_{\mathrm{L}}\right)$ and (f) the figure of merit $(\mathrm{zT})$ of $\mathrm{S}_{\mathrm{x}} \mathrm{Co}_{4} \mathrm{Sb}_{12}$ as function of temperature.

\section{CONCLUSIONS}

The thermoelectric properties of electronegative guest sulphur filled $\mathrm{Co}_{4} \mathrm{Sb}_{12}$ were investigated. Sulphur was successfully incorporated into the voids through the FFL was low ( 0.04$)$ verified using EPMA. S and $\rho$ decreased with increasing sulphur content due to increased $\mathrm{n}$ verified by Hall measurement. The P.F of filled samples enhanced. $\kappa$ decreased in filled samples compared to the pristine sample due to increased phonon scattering. $P$-Type filled skutterudite with highest zT of 0.18 at $623 \mathrm{~K}$ for $\mathrm{S}_{0.15} \mathrm{Co}_{4} \mathrm{Sb}_{12}$ was achieved due to low $\rho$ and $\kappa$.

\section{ACKNOWLEDGEMENTS}

RCM would like to thank Brain Pool Foundation, South Korea for providing the Brain Pool invited scientist fellowship (Project No: 172S-2-3-1967) to carry out the research work at Kongju National University, Republic of Korea.

\section{REFERENCES}

1. J. W. Sharp et. al. Journal of Applied Physics 78(2), 1013-1018 (1995).

2. Itoh T, Hattori E and Kitagawa K, Journal of Propulsion and Power. 24, 359-64 (2008).

3. X. Shi, W. Zhang, L. D. Chen and J. Yang, Physical Review Letters 95(18), 185503 (2005).

4. B. Duan et. al. Energy and Environmental Science 9(6), 2090-2098 (2016).

5. S. Wan at. el. ACS Applied Materials and Interfaces 10(1), 625-634 (2017).

6. X. Li at. el. Journal of Alloys and Compounds 615, 177-180 (2014).

7. H. J. Goldsmid and J. W. Sharp, J. Electron. Mater. 28, 869-872 (1999) 